EPJ Web of Conferences 116, 03006 (2016)

DOI: $10.1051 /$ epjconf/201611603006

(C) Owned by the authors, published by EDP Sciences, 2016

\title{
Characterisation and testing of the KM3NeT acoustic positioning system
}

\author{
S. Viola ${ }^{1, a}$, F. Simeone ${ }^{2, b}$, and M. Saldaña ${ }^{3, c}$ for the KM3NeT Collaboration \\ ${ }^{1}$ Istituto Nazionale di Fisica Nucleare, Laboratori Nazionali del Sud \\ ${ }^{2}$ Istituto Nazionale di Fisica Nucleare, Sezione di Roma \\ ${ }^{3}$ Instituto de Investigación para la Gestión Integrada de las Zonas Costeras, Universitat Politècnica \\ de València, Gandia, Spain
}

\begin{abstract}
In underwater neutrino telescopes, the search of point-like sources through the Cherenkov detection technique requires a precise knowledge of the positions of thousands of optical sensors, spread in a volume of a few cubic kilometres. In KM3NeT the optical sensors are hosted in $700 \mathrm{~m}$ high semi-rigid structures, called detection units, which move under the effects of underwater currents. These movements are continuously monitored through an underwater positioning system based on acoustic emitters and receivers. In this work, the tests performed on the key elements of the positioning system are presented.
\end{abstract}

\section{Introduction}

The KM3NeT neutrino telescope has been designed to measure the visible Cherenkov photons originated by charged particles propagating at velocities greater than the speed of light through the seawater by means of an array of photomultipliers (PMTs). The charged particle tracks are reconstructed measuring the time of arrival of the Cherenkov photons on PMTs, placed at known positions. In each detection unit (DU) of the telescope, PMTs are located within 18 digital optical modules (DOMs), that host 31 PMTs each one [1]. The position of each DOM is calculated acoustically by means of a Long Base Line of acoustic beacons placed in fixed positions and an array of digital acoustic receivers (DARs) installed in each DOM and at the base of the DUs and of the calibration units (CUs) [2].

\section{LBL acoustic beacon characterization}

The acoustic beacon, used for the KM3NeT acoustic positioning system is composed by a piezo-ceramic transducer and an electronics board integrated in an cylindrical hard-anodized aluminium vessel by the MSM company ([3]). The transducer is a Free Flooded Ring (FFR SX30) manufactured by Sensor Technology Ltd [4]. The electronic board is specifically designed to fulfil the requirements of the

\footnotetext{
ae-mail: sviola@lns.infn.it

b e-mail: francesco.simeone@roma1.infn.it

c e-mail: masalcos@upv.es
}

This is an Open Access article distributed under the terms of the Creative Commons Attribution License 4.0, which permits unrestricted use, distribution, and reproduction in any medium, provided the original work is properly cited. 


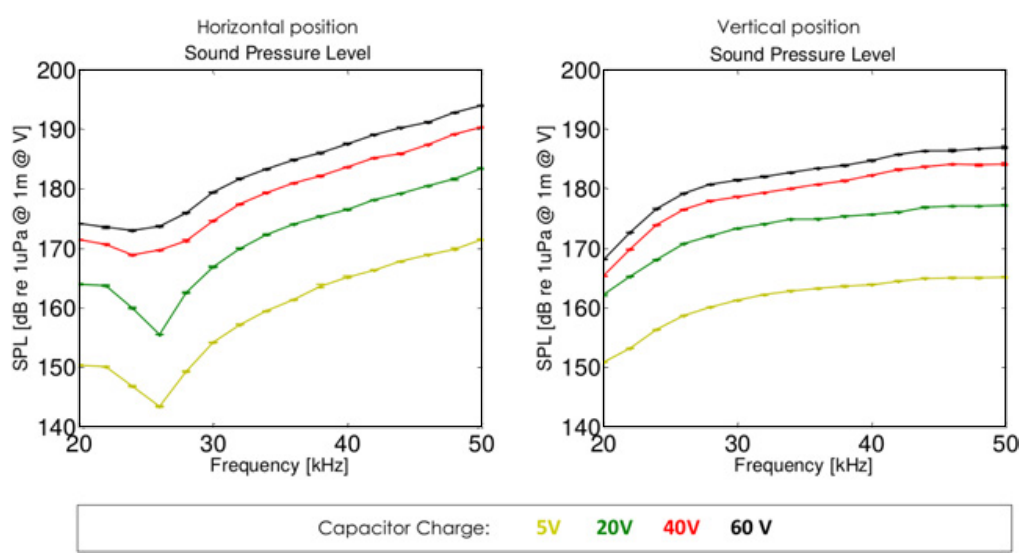

Figure 1. Sound Pressure Level (SPL) of an acoustic beacon at axial direction (left side) and radial direction (right side) for different capacitor charges.

telescope positioning system, enabling the transducer communication and the signal emission control and amplification [5]. The acoustic signal emission parameters can be configured by commands through a RS-232 serial interface allowing signal reconfiguration from shore. The acoustic beacon (MAB-100) for the LBL positioning system is a broadband range acoustic emitter $(20 \mathrm{kHz}-60 \mathrm{kHz})$ able to work at rating depths up to 400 bar in underwater environments. It provides the emission of short intense signals (Sound Pressure Levels of $180 \mathrm{~dB}$ re $1 \mu \mathrm{Pa}$ at $1 \mathrm{~m}$ at $34 \mathrm{kHz}$ ) with a variation of $\pm 6 \mathrm{~dB}$ in the frequency range of $20 \mathrm{kHz}$ to $60 \mathrm{kHz}$. The radial beam pattern is omnidirectional with $\pm 2 \mathrm{~dB}$ for each work frequency and the axial beam pattern is toroidal with $\pm 10 \mathrm{~dB}$ of variation at $60^{\circ}$ and $\pm 5 \mathrm{~dB}$ at $180^{\circ}$. The signal emission is activated by responding to an external trigger, in addition, it has two operation emission modes: automatic (continue) and triggered emission. The electronics delay of the object, that is the time from the reception of the trigger to the acoustic signal emission, is provided by the manufacturer. Typical values are $4.2 \pm 0.8 \mu \mathrm{s}$. The Acoustic Beacons were characterized in a water tank of $87.5 \times 113 \times 56.5 \mathrm{~cm}^{3}$ with fresh-water. The calibration was performed in terms of measuring the Transmitting Voltage Response (TVR) and directivity in emission. During the calibration, the distance between emitter (Acoustic Beacon) and receiver (calibrated hydrophone) was fixed at $20 \mathrm{~cm}$. The signals sent for the calibration were tone bursts of 5 wavelength cycles with frequencies ranging from $20 \mathrm{kHz}$ to $60 \mathrm{kHz}$ in steps of $2 \mathrm{kHz}$. Measurements were performed with different capacitor charge $(5 \mathrm{~V}, 20 \mathrm{~V}$, $40 \mathrm{~V}$ and $60 \mathrm{~V}$ ) both in radial and axial direction (Fig. 1). The hydrophone used for the calibration was the omnidirectional transducer model ITC-1042 [7]. The signal generation and acquisition process was conducted by a National Instruments PCI-Technology controller NI8176, which also controlled the positioning [6].

\section{Digital Acoustic Receivers characterisation}

In the KM3NeT acoustic positioning system the acoustic signals produced by the acoustic emitters of the LBL are received by two kinds of acoustic receivers: an "internal" digital piezoelectric-sensor, installed inside the DOM and a digital "external" hydrophone, installed at the base of the DUs and on the CUs, where no DOM are forseen. 


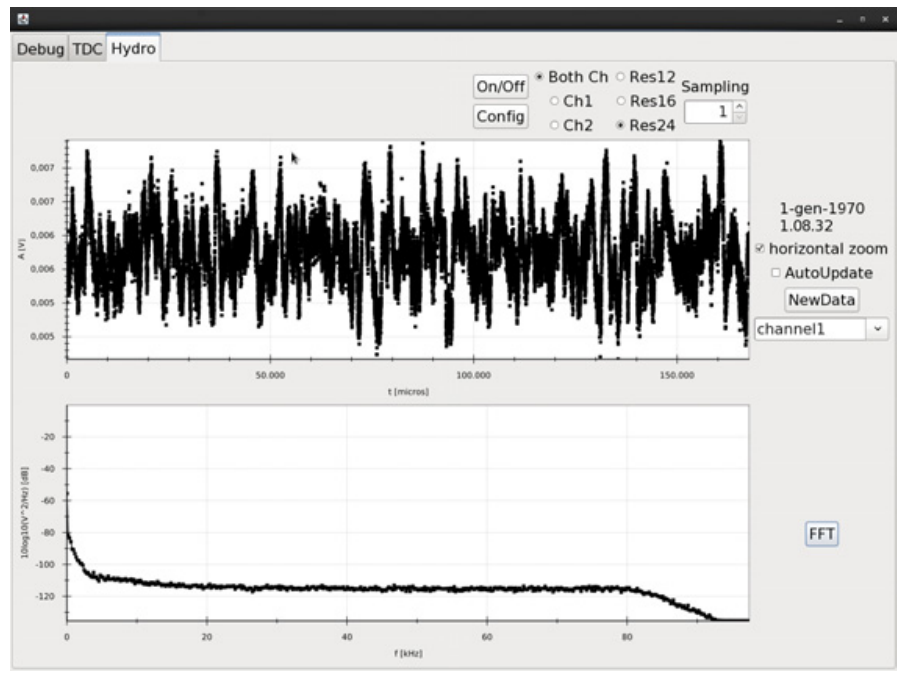

Figure 2. Oscillogram and power spectral density of the electronic noise of a typical "internal" digital acoustic receiver.

\subsection{The internal Digital Acoustic Receiver}

The "internal" Digital Acoustic Receiver is a digital piezoelectric-sensor based on a ceramic material produced by Ferroperm [8], readout by 24-bit Sigma-Delta ADC (model AD7764BRUZ -TSSOP28) [9]. The sensor is glued to the inside to the glass sphere of each DOM. The performance of the digital piezo sensors were investigated in the laboratory. Power spectral density of the electronic noise was measured thanks to a proper GUI (see Fig. 2) that provides in real time spectrum and oscillogram of the acquired signal. The noise spectrum shows a very flat behaviour in the range $10 \mathrm{kHz}$ to $80 \mathrm{kHz}$. Beyond that frequency the anti-alias filter sets in. Below $\approx 5 \mathrm{kHz}$ a strong rise of the noise can be observed before the effect of a high pass filter, which is intended to prevent saturation of the sensor due to strong low frequency noise. The RMS of the noise was measured to be about $1250 \mathrm{LSB}\left(1 \mathrm{LSB}=4 \mathrm{~V} / 2^{24}\right)$ for the full range and about $300 \mathrm{LSB}$ for the frequency range above $5 \mathrm{kHz}$. A test with the final configuration for DU-1 of KM3NeT was performed at Nikhef laboratory with an open DOM inside a light-tight box. It was observed that turning on the PMT power results in spikes at distinct frequencies. However, in the range of frequencies between 20 and $40 \mathrm{kHz}$ where the positioning system will be operating, no disturbances are observed so that no adverse effects on the performance of the position reconstruction are expected. The sensor's functionality check is also performed after installation in the DOM, during the final DOM calibration measurement. A function generator produces sinusoidal signals at arbitrary amplitude (always the same for all DOM tests) at $30 \mathrm{kHz}$ frequency. The electric signal is amplified by an audio amplifier and fed into a piezo-electric, not-magnetic tweeter, placed close to the piezo-sensor. One minute of continuous data taking is analysed on-line looking at noise spectrum the presence of the peak produced by the emitter.

\subsection{The external acoustic Digital receivers: Hydrophones}

External hydrophones are hosted on the base of the DUs and on CUs, where no DOM is foreseen. These hydrophones will be used to calibrate the LBL, measuring the relative distances among LBL elements. The selected hydrophone is the DG0330 manufactured by CO.L.MAR. s.r.l. [10]. It consists of a spherical piezo-ceramic element, read-out by an analogue board splitting the signal in two lines 
with different gains $(+46 \mathrm{~dB}$ and $+26 \mathrm{~dB}$ respectively). The double gain feature was implemented to avoid signal saturation when the hydrophone is placed close (about $1 \mathrm{~m}$ ) to the beacon. Two hydrophone productions are available, without and with an analogue signal high-pass filtering stage. The filter frequency is $700 \mathrm{~Hz}$, to reject the low frequency ambient sea-noise and thus improve Signal to Noise ratio in the detection of beacon pulses $(20-40 \mathrm{kHz})$. The unfiltered version can be also used for acoustic noise monitoring and marine bio-acoustics. The hydrophones are delivered from the company after a pressure-crush test at 400 bars (4000 m water equivalent) and shallow water calibration (at 1 bar). A sub batch of few hydrophones was also calibrated at high pressure (350 bars). Once received at INFN, the hydrophones were tested with a dedicated set-up, current and intrinsic noise were measured for the two channels using proprietary software. The time response of the hydrophone as a function of the frequency was measured and reported in acceptance test sheets. A functional test (communication and noise spectrum visualization) is carried out before the closure of the DU Base container and line installation. For all hydrophones the sensitivity curves and the time response were stored in the database.

\section{Conclusions}

Measurements in the water tank demonstrate that the acoustic pulses produced by the acoustic beacon can reach a SPL of $180 \mathrm{~dB}$ re $1 \mu \mathrm{Pa}$ in the range of frequencies from $20 \mathrm{kHz}$ to $50 \mathrm{kHz}$. It means that beacon signals in this range can be easily recognised by the acoustic receivers of the telescope since that an acoustic pulse with a SPL of $180 \mathrm{~dB}$ re $1 \mu \mathrm{Pa}$ at $1 \mathrm{~m}$ can effectively propagate in water up to distance of $2 \mathrm{~km}$ with about $110 \mathrm{~dB}$ re $1 \mu \mathrm{Pa}$ at $0^{\circ}$ (depending on the frequency). "Internal" acoustic receiver functionality in deep sea was demonstrated analysing acoustic data after the deployment of the first detection unit at the KM3NeT-IT site. Sweep sine signals from $38 \mathrm{kHz}$ to $39 \mathrm{kHz}$, emitted by a LBL beacon (capacitor charge $50 \mathrm{~V}$ ) have been used as test signals for the receivers located along the DU at distances between $220 \mathrm{~m}$ and $690 \mathrm{~m}$ from the beacon. The data analysis showed that beacon pulses were properly detected by all the acoustic receivers.

\section{References}

[1] KM3NeT website, www.km3net.org

[2] S.Viola , Proceedings of $34^{\text {th }}$ ICRC, PoS(ICRC2015)1169 (2015)

[3] Mediterráneo Señales Marítimas (MSM) website, http://en.mesemar.com/

[4] Sensor Technology std website: http://sensortech.ca

[5] C D Llorens et al., Journal of Instrumentation, 7, C01001 (2012)

[6] PXI-8176 Controller website, http://sine.ni.com/nips/cds/view/p/lang/it/nid/ 212342

[7] ITC hydrophone data sheet, http://www.channeltechgroup.com/publication/view/ model-itc-1042-spherical-omnidirectional-transducer

[8] FERROPERM website, www . ferroperm-piezo.com

[9] Analog Devices website, www . analog.com

[10] CO.L.MAR. srl website, www. colmaritalia.it 